LA-14432

Approved for public release;

distribution is unlimited.

Continuous Wave Laser Irradiation of

Explosives

- Los Alamos 
Los Alamos National Laboratory, an affirmative action/ equal opportunity employer, is operated by Los Alamos National Security, LLC, for the National Nuclear Security Administration of the U.S. Department of Energy under contract DE-AC52-06NA25396.

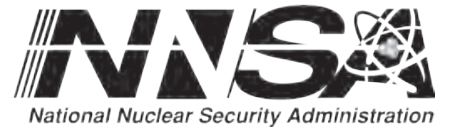

National Nuclear Security Administration

This report was prepared as an account of work sponsored by an agency of the U.S. Government. Neither Los Alamos National Security, LLC, the U.S. Government nor any agency thereof, nor any of their employees make any warranty, express or implied, or assume any legal liability or responsibility for the accuracy, completeness, or usefulness of any information, apparatus, product, or process disclosed, or represent that its use would not infringe privately owned rights. Reference herein to any specific commercial product, process, or service by trade name, trademark, manufacturer, or otherwise does not necessarily constitute or imply its endorsement, recommendation, or favoring by Los Alamos National Security, LLC, the U.S. Government, or any agency thereof. The views and opinions of authors expressed herein do not necessarily state or reflect those of Los Alamos National Security, LLC, the U.S. Government, or any agency thereof. Los Alamos National Laboratory strongly supports academic freedom and a researcher's right to publish; as an institution, however, the Laboratory does not endorse the viewpoint of a publication or guarantee its technical correctness. 
LA-14432

Issued: December 2010

Continuous Wave Laser Irradiation of

Explosives

Shawn D. McGrane

David S. Moore

Los Alamos 



\title{
Continuous Wave Laser Irradiation of Explosives
}

by

\author{
Shawn D. McGrane and David S. Moore
}

\begin{abstract}
Quantitative measurements of the levels of continuous wave (CW)

laser light that can be safely applied to bare explosives during contact operations were obtained at $532 \mathrm{~nm}, 785 \mathrm{~nm}$, and $1550 \mathrm{~nm}$ wavelengths. A thermal camera was used to record the temperature of explosive pressed pellets and single crystals while they were irradiated using a measured laser power and laser spot size. A visible light image of the sample surface was obtained before and after the laser irradiation. Laser irradiation thresholds were obtained for the onset of any visible change to the explosive sample and for the onset of any visible chemical reaction. Deflagration to detonation transitions were not observed using any of these $\mathrm{CW}$ laser wavelengths on single crystals or pressed pellets in the unconfined geometry tested. Except for the photochemistry of DAAF, TATB and PBX 9502, all reactions appeared to be thermal using a $532 \mathrm{~nm}$ wavelength laser. For a $1550 \mathrm{~nm}$ wavelength laser, no photochemistry was evident, but the laser power thresholds for thermal damage in some of the materials were significantly lower than for the $532 \mathrm{~nm}$ laser wavelength. No reactions were observed in any of the studied explosives using the available $300 \mathrm{~mW}$ laser at $785 \mathrm{~nm}$ wavelength. Tables of laser irradiance damage and reaction thresholds are presented for pressed pellets of PBX9501, PBX9502, Composition B, HMX, TATB, RDX, DAAF, PETN, and TNT and single crystals of RDX, HMX, and PETN for each of the laser wavelengths.
\end{abstract}




\section{Introduction}

There have been many questions, especially recently because of the widespread use of interferometric detonation- and/or shock-velocity diagnostics that utilize high power $532 \mathrm{~nm}$ or $1550 \mathrm{~nm}$ lasers, regarding the levels of laser light that can be safely applied to bare explosives during contact operations. While several studies have looked at certain types-of-explosive/types-of-laser combinations, it is still often difficult or impossible to make informed safety decisions. Indeed, there have been a large number of studies of laser ignition of explosives using $\mathrm{CO}_{2}$ lasers, where the $10 \mu \mathrm{m}$ infrared laser wavelength is strongly absorbed in the samples (for example, see References 1-6). There is a much different situation for the visible and near-infrared laser wavelengths of concern for this study.

We therefore undertook the quantitative study of continuous wave $(\mathrm{CW})$ lasers on several types of explosives at three wavelengths: $532 \mathrm{~nm}, 785 \mathrm{~nm}$, and $1550 \mathrm{~nm}$. The $532 \mathrm{~nm}$ wavelength is commonly used as a diagnostic in VISAR. A large number or portable Raman spectroscopic devices utilize a high power $785 \mathrm{~nm}$ diode laser. The new interferometric shock velocity diagnostic called "photon Doppler velocimetry" or "heterodyne velocimetry" (PDV or Het-V) utilizes optical fiber lasers at wavelengths near $1550 \mathrm{~nm}$. Results from a previous study at Lawrence Livermore National Laboratory (LLNL) using a $1550 \mathrm{~nm}$ laser are compared to our results. ${ }^{7}$ Significant data already exists for nanosecond pulsed laser detonation thresholds at multiple wavelengths; ${ }^{8}$ however, there are a variety of complicated issues involved with pulsed laser interactions with materials. The situation is much simpler for $\mathrm{CW}$ irradiation, so we have chosen to begin this in-depth quantitative study of laser irradiation of explosives using $\mathrm{CW}$ lasers.

The experiments reported here are simple. We record the temperature of an explosive sample with a thermal camera at 10 frames per second for 120 seconds while the sample is irradiated by the laser. We record a visible light image of the surface of the explosive sample before and after laser irradiation. We begin with a low irradiance (power/area) and subsequently increase the irradiance in discrete steps of increasing power. Any reactions are noted. If there is a reaction, a fresh sample region is used for the next irradiance level. Reaction does not imply a safety-significant event but a rather a material change: melting TNT is a reaction, but it does not necessarily present a hazard.

The data and results presented here are preliminary. Most of the samples are pressed powders with significant light scattering. Therefore, the irradiated volume is not precisely known. The laser power actually absorbed by a sample is usually determined by an absorptance measured using the Beer-Lambert law. For highly scattering materials, the situation is complicated by the unknown sample absorption length caused by multiple scattering events. Radiation transfer theory and Monte Carlo simulations have been used to estimate the scattering length for turbid samples such as body tissue, but we could find no measurements of the scattering cross section for these explosives samples. 


\section{Experimental Details}

The $532 \mathrm{~nm}$ excitation was performed with a Spectra Physics Millenia Nd:YLF laser, $\mathrm{CW}$, intracavity frequency doubled to $532 \mathrm{~nm}$. Power was set to 1 to $3000 \mathrm{~mW}$ at the sample. The laser was focused with a 6 " focal length achromat to a spot size of $230 \times 245 \mu \mathrm{m}$ on the sample surface $\left(\right.$ area $=4.42 \times 10^{-4} \mathrm{~cm}^{2}$ ). The laser powers were measured with a calibrated ThorLabs Model PM100A power meter with ThorLabs S310C head for high powers and S210C head for low powers.

The $1550 \mathrm{~nm}$ excitation was performed with an IPG Fiber Laser (model ELR-5-1550$\mathrm{SF})$. The optical fiber output was focused onto the surface of the pressed pellet explosives samples using a $20 \mathrm{~mm}$ focal length singlet lens to a spot size of $230 \times 315 \mu \mathrm{m}$ (area $=$ $\left.7.25 \times 10^{-4} \mathrm{~cm}^{2}\right)$.

The $785 \mathrm{~nm}$ excitation was performed using an Ahura First Defender portable Raman device. The scan settings were set to manual, 10 -second accumulation, which was measured to be actually 15 -second exposure (rather than the 120 seconds for the other two laser wavelengths discussed above). We do not yet have access to a high power 785 $\mathrm{nm}$ laser diode to allow longer exposures, but the data presented below show that, for the samples measured in this report, this is not a major issue. The focal spot size on the sample surface was measured to be $140 \times 190 \mu \mathrm{m}\left(\right.$ area $\left.=2.66 \times 10^{-4} \mathrm{~cm}^{2}\right)$. Only the "high" power level was utilized, which was $299 \mathrm{~mW}$.

Visible imaging used a ThorLabs Model DCC1545M USB/CCD camera through a Tamron $75 \mathrm{~mm}$ fl CCD camera lens mounted on a 4" extension tube to provide a $4.8 \mathrm{~mm} \times 3.8 \mathrm{~mm}$ field of view (FOV) at the full image size, which was $1280 \times 1024$ pixels.

Thermal imaging was performed with a FLIR ThermoVision SC4000 camera with 3-5 $\mu \mathrm{m}$ band Indium Antimony ( $\mathrm{InSb}$ ) focal plane array through a germanium $100 \mathrm{~mm} \mathrm{fl}$ telephoto lens mounted on a 2" extension tube to provide an FOV of $20 \times 16 \mathrm{~mm}$ at the full image size, which was $320 \times 256$ pixels. The temperature was calibrated from $23^{\circ} \mathrm{C}$ to $300^{\circ} \mathrm{C}$ using a calibrated blackbody source (Mikron M316) and nonlinear nonuniformity correction applied to the data. The $3 \mathrm{~mm}$ diameter samples were ca. 50 pixels in diameter, and the ca. $250 \mu \mathrm{m}$ diameter laser focus was about 4 pixels in diameter.

The explosive samples were mounted in or on (for crystals and $785 \mathrm{~nm}$ excitation) a 4" diameter block of PMMA in which nine $3 \mathrm{~mm}$ diameter $\times 4 \mathrm{~mm}$ deep holes were bored. Explosive samples were pressed to $3 \mathrm{~mm}$ diameter by ca. 3-4 $\mathrm{mm}$ long right circular cylinders (50 mg mass) using a 15 ton pneumatic press ( 1 minute at 60,000 psi). These pressed samples have densities of mostly $>95 \%$ TMD. No window was used to provide pressure confinement or to provide a thermal conductive interface. Single crystals of explosives were simply set on the PMMA block. A blast shield surrounded the samples to protect the operators and equipment. The experimental arrangement without the blast shield is shown in Figure 1. 


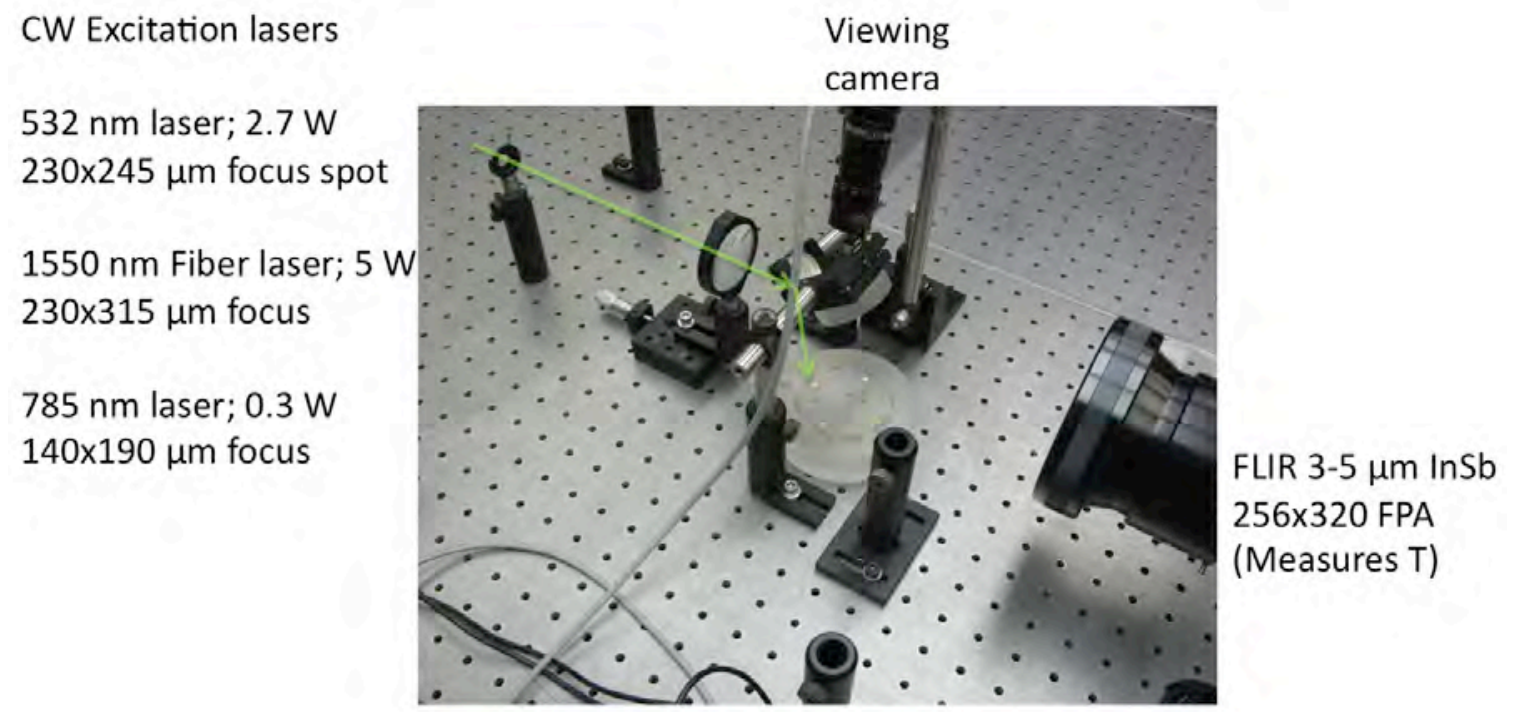

Figure 1. Experimental arrangement. The laser comes in from the left (parameters are listed). Visible imaging is from above, and thermal imaging is at right. Explosive samples in cylindrical PMMA block are at the center.

\section{2 nm Results}

Single crystals

Laser heating data from single crystals of PETN, RDX, and HMX are shown in Figure 2. Even at $2500 \mathrm{~mW}$, the maximum heating was only about $22^{\circ} \mathrm{C}$ above ambient. The temperature stabilized at long times with no transient temperature excursions. There was no visible change in any of the crystals at the maximum power employed, which is not surprising, considering the minimal absorption and therefore minimal heating. The crystals were monitored during irradiation through neutral density filters. There was no apparent safety risk in applying $532 \mathrm{~nm} \mathrm{CW}$ light up to $2500 \mathrm{~mW}$ to single crystals of PETN, RDX, or HMX. 

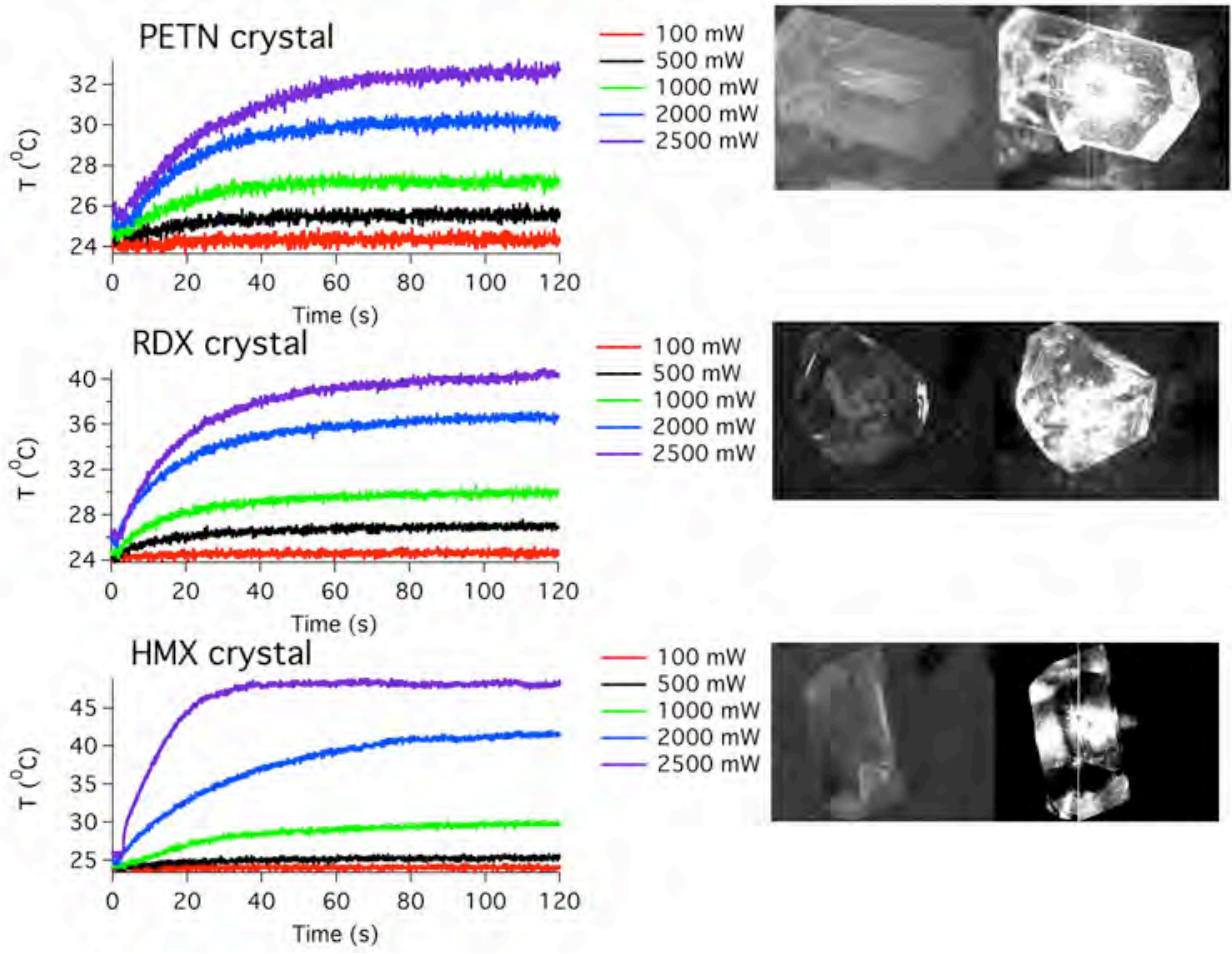

Figure 2. Left: Temperature versus time at the center of a laser spot, single pixel. Right: visible image of crystal before (left) and during (right) laser irradiation. The after irradiation image matched the before irradiation image.

This data can be summarized as in Figure 3, which shows the maximum temperature reached after 120 seconds of irradiation vs laser power for each material.

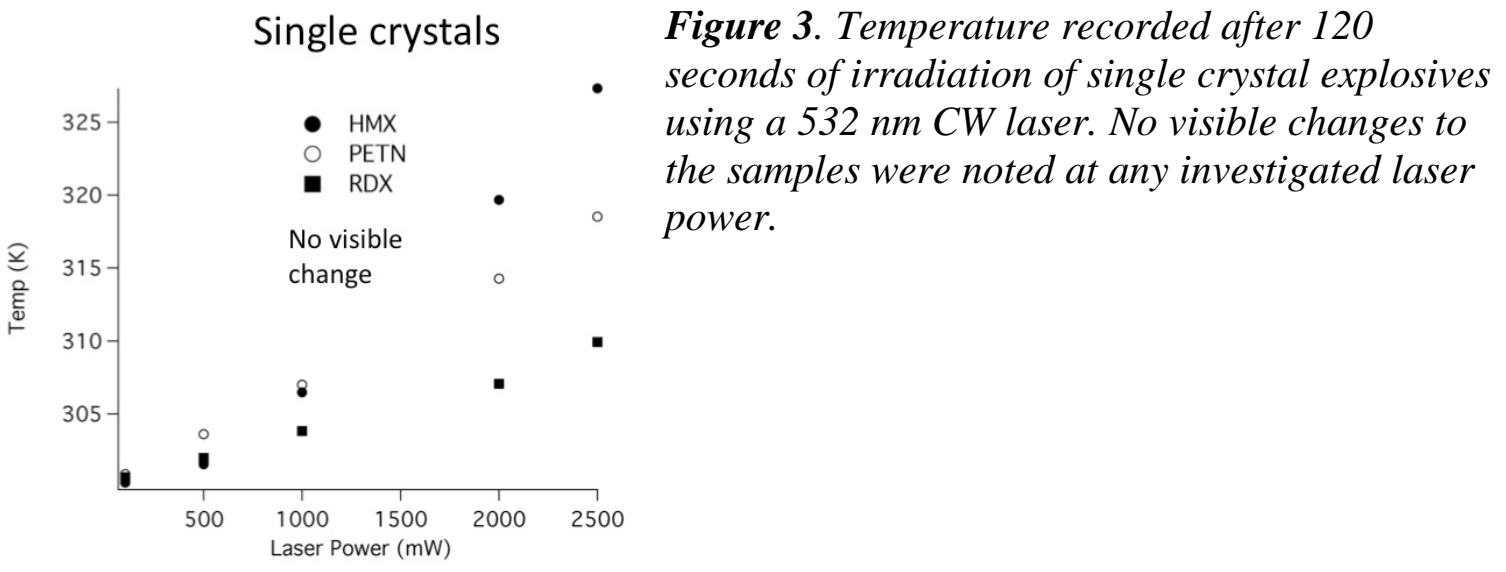


The $532 \mathrm{~nm}$ laser (CW) irradiation data for pressed pellets of these explosives can be summarized as in Figure 4.
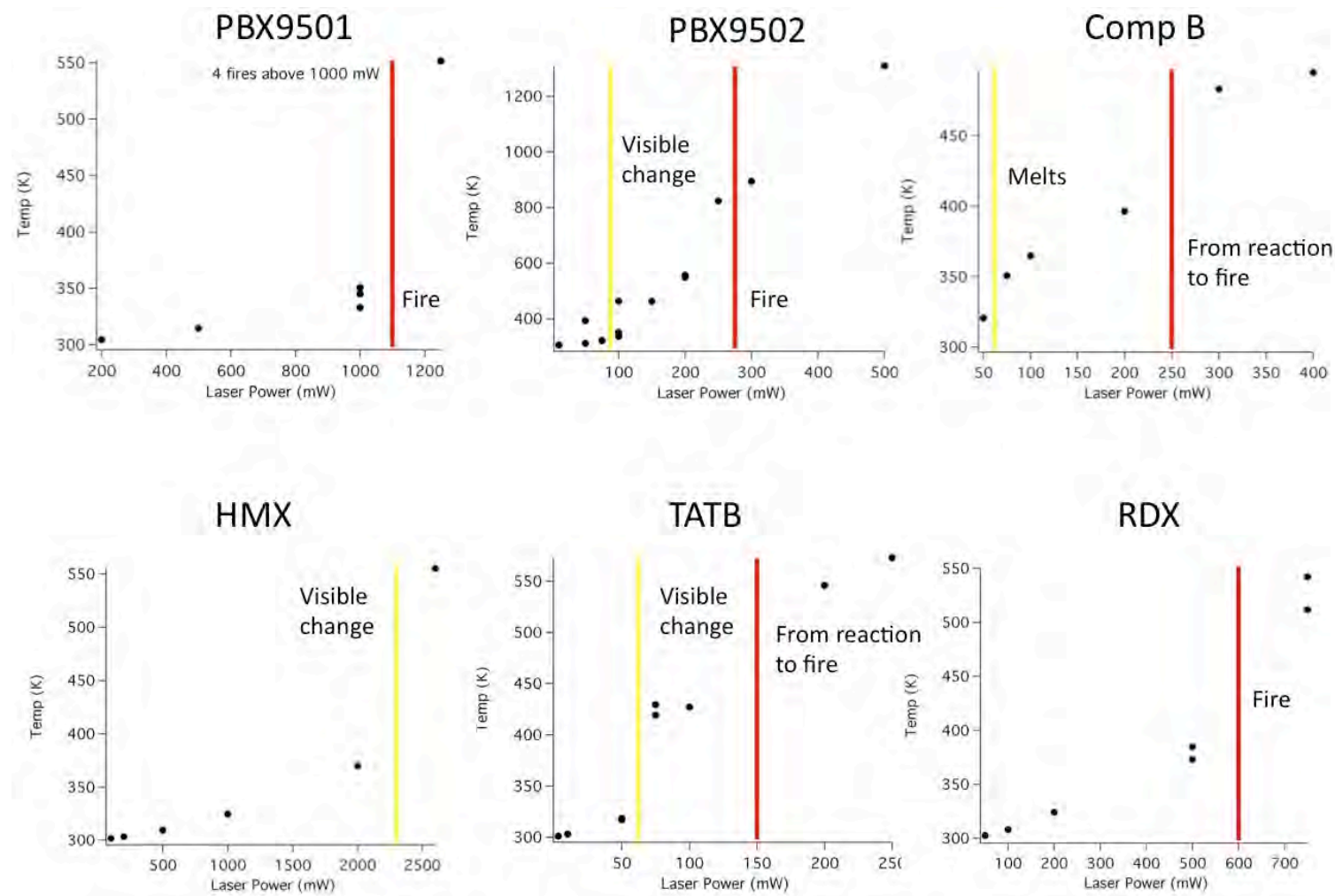

DAAF

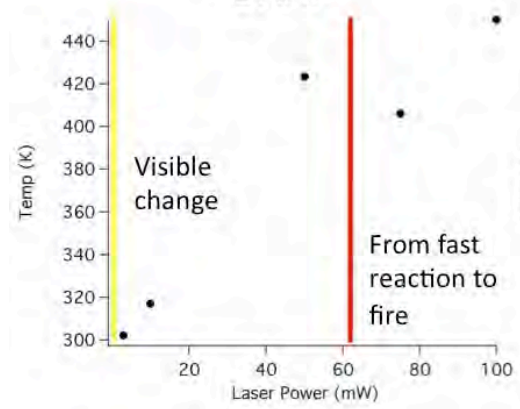

PETN

TNT
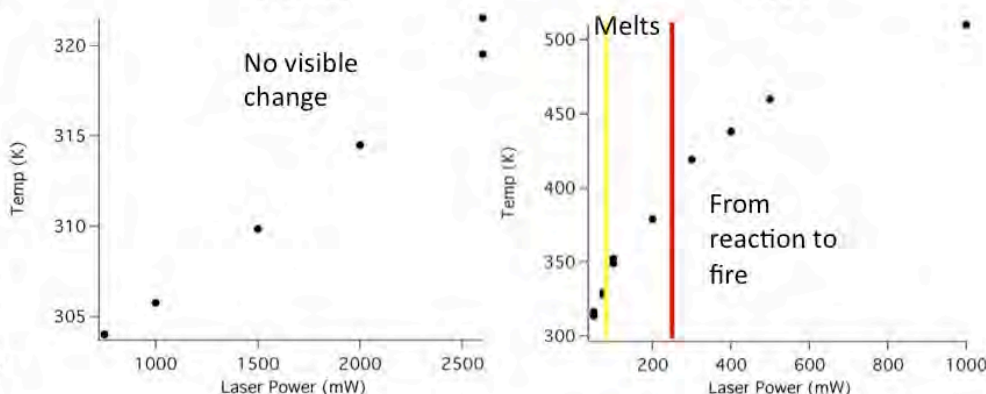

Figure 4. Maximum temperature at the illuminated spot of each pressed explosive sample during 120 seconds of irradiation (532 $\mathrm{nm}$ laser) vs laser power. Yellow vertical lines denote the threshold (irradiance values are shown in the table below) for visible change or phase transition (e.g., melting) but no chemical reaction. The red vertical lines denote the threshold for visible chemical reaction such as smoke and fire (usually accompanied by large abrupt changes in FLIR measured temperature).

Table 1 presents the power thresholds for visible change or melting and visible chemical reaction for the explosives studied using CW $532 \mathrm{~nm}$ laser light. 
Table 1. Visible Change and Visible Chemical Reaction Laser Irradiance Thresholds for Several Explosives (Pressed Pellets) for $532 \mathrm{~nm}$ CW Lasers

\begin{tabular}{|l|l|l|}
\hline Explosive & Visible Change Threshold & Reaction Threshold \\
\hline PBX9501 & None observed & $2260 \mathrm{~W} / \mathrm{cm}^{2}$ \\
\hline PBX9502 & $204 \mathrm{~W} / \mathrm{cm}^{2}$ & $610 \mathrm{~W} / \mathrm{cm}^{2}$ \\
\hline Composition B & $136 \mathrm{~W} / \mathrm{cm}^{2}$ (melting) & $565 \mathrm{~W} / \mathrm{cm}^{2}$ \\
\hline HMX & $>4500 \mathrm{~W} / \mathrm{cm}^{2}$ & $>5600 \mathrm{~W} / \mathrm{cm}^{2}$ \\
\hline TATB & $136 \mathrm{~W} / \mathrm{cm}^{2}$ (surface browns) & $340 \mathrm{~W} / \mathrm{cm}^{2}$ \\
\hline RDX & None observed & $1360 \mathrm{~W} / \mathrm{cm}^{2}$ \\
\hline DAAF & $2.5 \mathrm{~W} / \mathrm{cm}^{2}$ (surface browns) & $136 \mathrm{~W} / \mathrm{cm}^{2}$ \\
\hline PETN & None observed & $>5600 \mathrm{~W} / \mathrm{cm}^{2}$ \\
\hline TNT & $180 \mathrm{~W} / \mathrm{cm}^{2}$ (melts) & $565 \mathrm{~W} / \mathrm{cm}^{2}$ \\
\hline
\end{tabular}

Visual images of samples damaged to varying degrees illustrate the typically localized heating and reaction that occurred, as shown in Figure 5.
9501
9502
Comp B
HMX
TATB

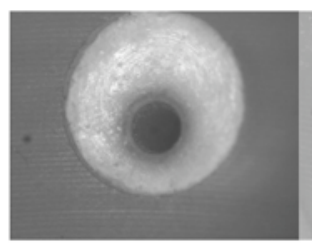

DAAF

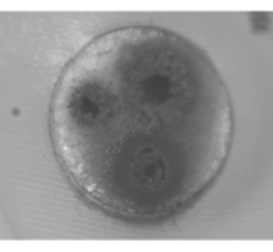

PETN

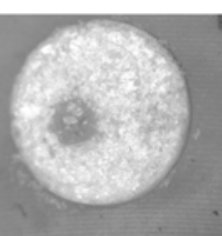

TNT

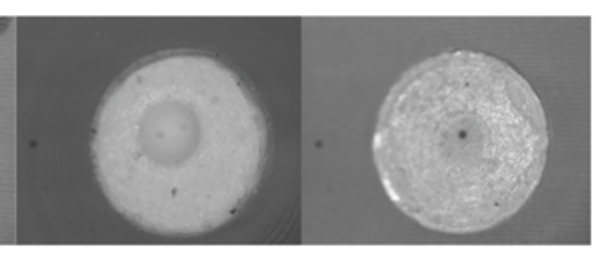

RDX
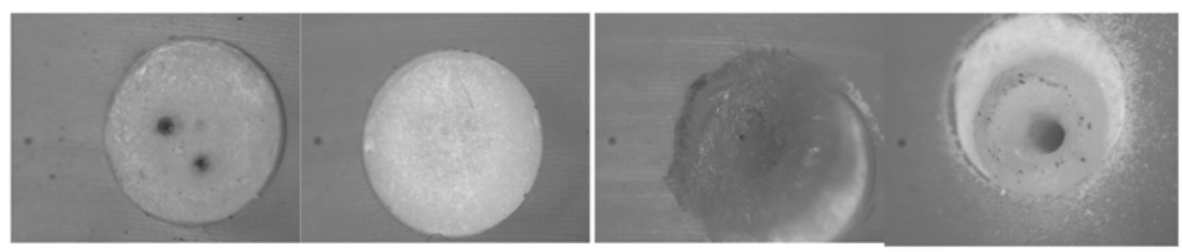

Figure 5. Visible light images of samples damaged from $532 \mathrm{~nm}$ irradiation at various power levels.

\section{$1550 \mathrm{~nm}$ Results}

Single crystals

Experiments performed at $1550 \mathrm{~nm}$ on single crystals showed substantially more energy absorption and heating. This wavelength is sufficiently close to vibrational overtone absorption resonances that the available power led to reactions, as shown in Figure 6. Each symbol in a given plot is a separate crystal, and some crystal-to-crystal variation was observed. This is likely due to the effect of random crystal orientation affecting the shape and intensity of the vibrational overtone absorption, but it was not carefully studied. While cracking will depend on crystal size and shape, it was the first reaction noted for the small crystals studied ( $<100 \mathrm{mg}$ ). Phase change in HMX crystals was clearly observed by crystal expansion and melting of localized pools of PETN and RDX 
concurrent with reaction. Crystals under $1550 \mathrm{~nm}$ laser irradiation that showed these effects are illustrated in Figure 7.
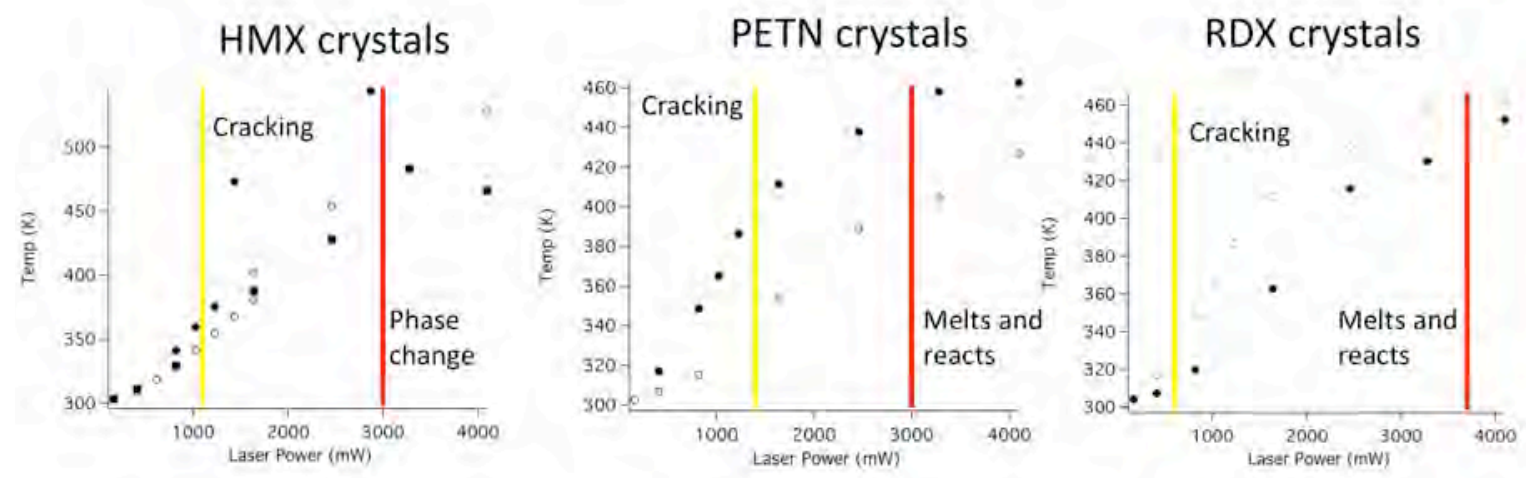

Figure 6. Maximum temperature recorded during 120 seconds of irradiation of single crystal explosives using a $1550 \mathrm{~nm}$ continuous wave laser. The yellow line marks the lowest power observed for a reaction of any sort, in these cases, cracking of the crystal. The red line marks chemical reaction, phase change, or fire, as noted.

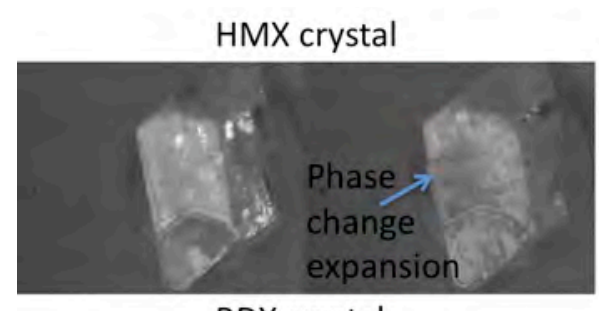

RDX crystal

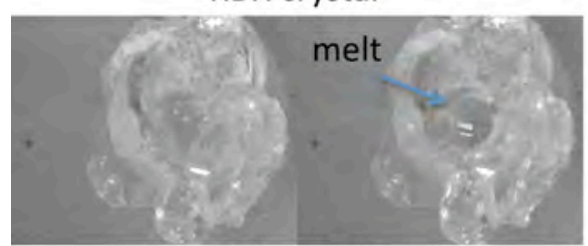

PETN crystals

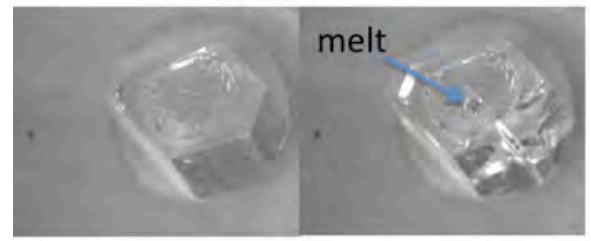

Figure 7. Single crystals under irradiation at $4100 \mathrm{~mW}$ of $1550 \mathrm{~nm}$. HMX showed a noticeable increase in volume near the irradiated spot, suggesting a $\beta \rightarrow \delta$ transition. $R D X$ and PETN melted and reacted in a round pool. All crystals cracked previously at lower powers.

Table 2 presents the power thresholds for visible change (cracking) and for melting or visible chemical reaction for PETN, RDX, and HMX single crystal explosives using CW $1550 \mathrm{~nm}$ laser light. Note the completely different behavior of these single crystal explosives when a $1550 \mathrm{~nm}$ laser is used as opposed to the behavior observed when a $532 \mathrm{~nm}$ laser is used (see Figure 3). 
Table 2. Laser Irradiance Thresholds $(1550 \mathrm{~nm})$ for Cracking and Observable Reaction in Single Crystal Explosives

\begin{tabular}{|l|l|l|}
\hline Explosive & Cracking Threshold & $\begin{array}{l}\text { Melt or Reaction } \\
\text { Threshold }\end{array}$ \\
\hline HMX (single crystal) & $2500 \mathrm{~W} / \mathrm{cm}^{2}$ & $6800 \mathrm{~W} / \mathrm{cm}^{2}$ \\
\hline PETN (single crystal) & $3200 \mathrm{~W} / \mathrm{cm}^{2}$ & $6800 \mathrm{~W} / \mathrm{cm}^{2}$ \\
\hline RDX (single crystal) & $1600 \mathrm{~W} / \mathrm{cm}^{2}$ & $8400 \mathrm{~W} / \mathrm{cm}^{2}$ \\
\hline
\end{tabular}

Pressed pellets

The $1550 \mathrm{~nm}$ laser $(\mathrm{CW})$ irradiation data for pressed pellets of these explosives can be summarized as in Figure 8.
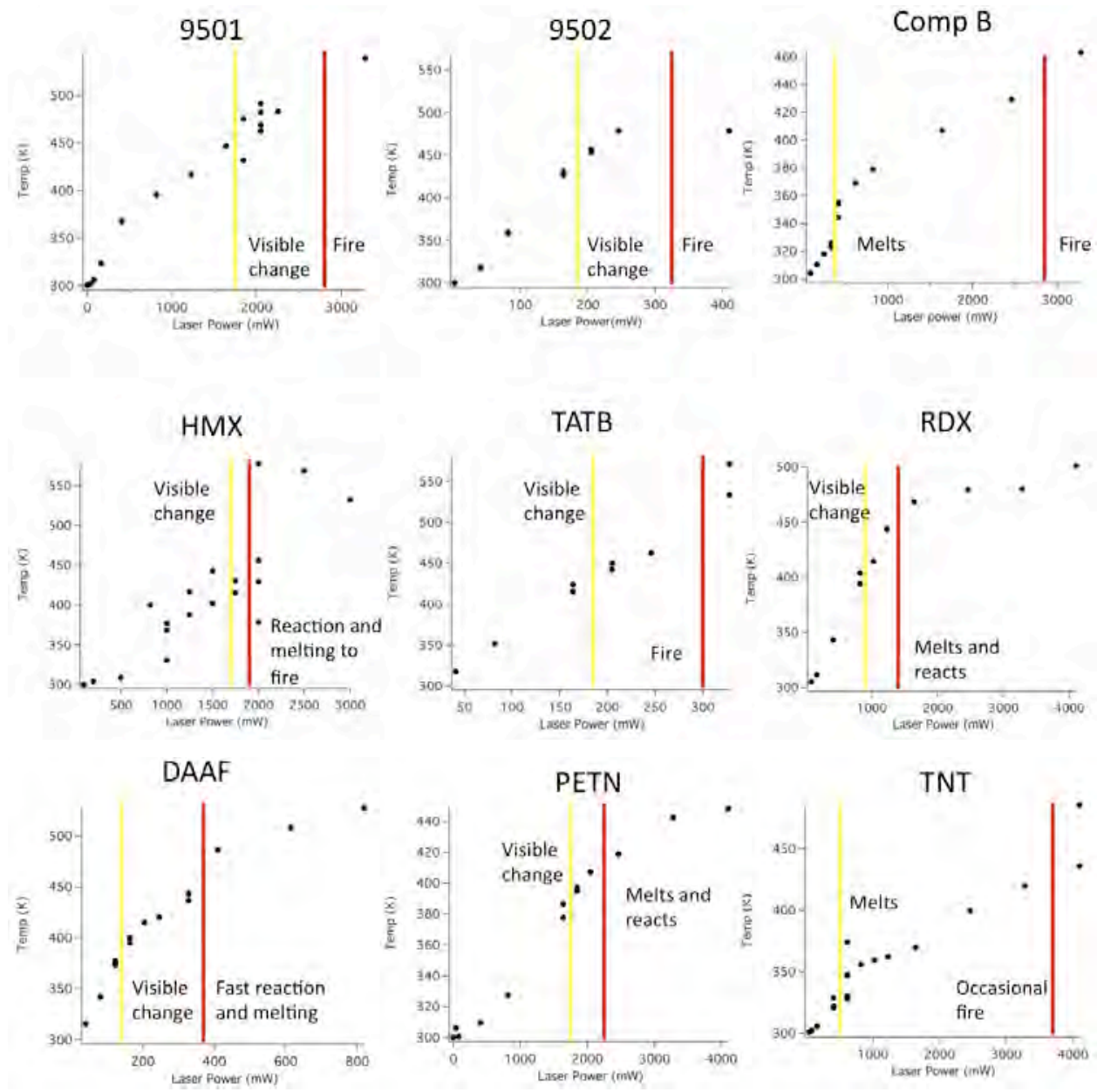

Figure 8. Maximum temperature at the illuminated spot of each pressed explosive sample during 120 seconds of irradiation (1550 nm laser) versus laser power. Yellow vertical lines denote the threshold (irradiance values are shown in Table 3) for visible change or phase transition (e.g., melting) but no chemical reaction. The red vertical lines denote the threshold for visible chemical reaction such as smoke and fire (usually accompanied by large abrupt changes in FLIR measured temperature). 
Table 3 presents the power thresholds for visible change or melting and visible chemical reaction for the explosives studied using CW $1550 \mathrm{~nm}$ laser light.

Table 3. Visible Change and Visible Chemical Reaction Laser Irradiance Thresholds for Several Explosives (Pressed Pellets) for $1550 \mathrm{~nm}$ CW Lasers

\begin{tabular}{|l|l|l|}
\hline Explosive (pressed) & Visible Change Threshold & Reaction Threshold \\
\hline PBX9501 & $4000 \mathrm{~W} / \mathrm{cm}^{2}$ & $6300 \mathrm{~W} / \mathrm{cm}^{2}$ \\
\hline PBX9502 & $400 \mathrm{~W} / \mathrm{cm}^{2}$ & $680 \mathrm{~W} / \mathrm{cm}^{2}$ \\
\hline Composition B & $900 \mathrm{~W} / \mathrm{cm}^{2}$ & $6300 \mathrm{~W} / \mathrm{cm}^{2}$ \\
\hline HMX & $3800 \mathrm{~W} / \mathrm{cm}^{2}$ & $4300 \mathrm{~W} / \mathrm{cm}^{2}$ \\
\hline TATB & $400 \mathrm{~W} / \mathrm{cm}^{2}$ & $680 \mathrm{~W} / \mathrm{cm}^{2}$ \\
\hline RDX & $2000 \mathrm{~W} / \mathrm{cm}^{2}$ & $3000 \mathrm{~W} / \mathrm{cm}^{2}$ \\
\hline DAAF & $340 \mathrm{~W} / \mathrm{cm}^{2}$ & $680 \mathrm{~W} / \mathrm{cm}^{2}$ \\
\hline PETN & $3800 \mathrm{~W} / \mathrm{cm}^{2}$ & $5000 \mathrm{~W} / \mathrm{cm}^{2}$ \\
\hline TNT & $1300 \mathrm{~W} / \mathrm{cm}^{2}(\mathrm{melts})$ & $8500 \mathrm{~W} / \mathrm{cm}^{2}$ \\
\hline
\end{tabular}

A previous study of laser damage using a $1550 \mathrm{~nm}$ fiber laser was undertaken at HEAF (Lawrence Livermore National Laboratory) by Dan Phillips and Paul Wilkins. Table 4 compares their data with ours for the explosives common to both studies. Note that the irradiated area in the LLNL experiments is not equal to their optical fiber diameter (as they assume in their reported threshold irradiances), but rather is determined by radiative transport within the highly scattering composite materials. Their irradiance thresholds are therefore overestimates. Similarly, our irradiance has not considered scattering. The LLNL experiments were also limited to $1470 \mathrm{~mW}$ total laser power.

Table 4. Comparison of Damage Threshold Laser Irradiance at $1550 \mathrm{~nm}$ to Reported Results (also at $1550 \mathrm{~nm}$ ) at HEAF (LLNL)

\begin{tabular}{|l|l|l|}
\hline Explosive & This Study Threshold & LLNL Threshold \\
\hline PBX9501 (LX-14) & $4000 \mathrm{~W} / \mathrm{cm}^{2}$ & $>2100000 \mathrm{~W} / \mathrm{cm}^{2}$ \\
\hline PBX9502 & $400 \mathrm{~W} / \mathrm{cm}^{2}$ & $540000 \mathrm{~W} / \mathrm{cm}^{2}$ \\
\hline Composition B & $900 \mathrm{~W} / \mathrm{cm}^{2}$ & $>2100000 \mathrm{~W} / \mathrm{cm}^{2}$ \\
\hline PETN & $3800 \mathrm{~W} / \mathrm{cm}^{2}$ & $1540000 \mathrm{~W} / \mathrm{cm}^{2}$ \\
\hline TATB & $4000 \mathrm{~mW} / \mathrm{cm}^{2}$ & $540000 \mathrm{~W} / \mathrm{cm}^{2}$ \\
\hline
\end{tabular}

The poor comparison of irradiance thresholds suggests that irradiance is not a good variable for comparison of safe power levels over dramatically different spot sizes ( $\sim 9 \mathrm{vs}$ $250 \mu \mathrm{m}$ radius) for these pressed powder samples. The volume over which the energy is absorbed is not limited by the spot size at the sample surface but depends on the scattering properties of the material. The caveat stated at the end of the Introduction is exactly applicable here.

Visual damage indicators typical of $1550 \mathrm{~nm}$ excitation are shown in Figure 9. 


\section{1
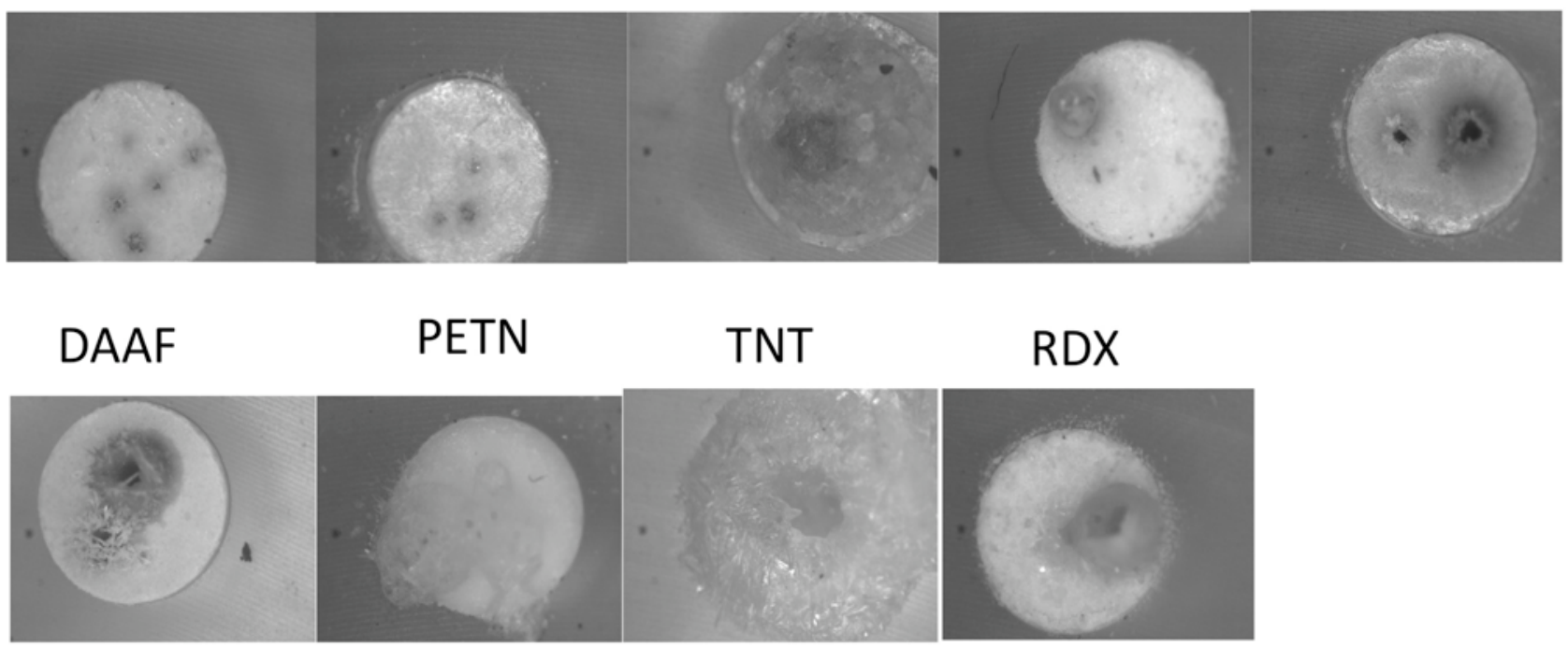

Figure 9. Visible light images of damage caused by $1550 \mathrm{~nm}$ irradiation at various power levels.

\section{$785 \mathrm{~nm}$ Results}

The temperature vs exposure time plots for the investigated materials are shown in Figure 10. Note that none of the samples rose above $330 \mathrm{~K}$ within the 15 -second exposure (longest exposure available using the Ahura First Defender portable Raman system).

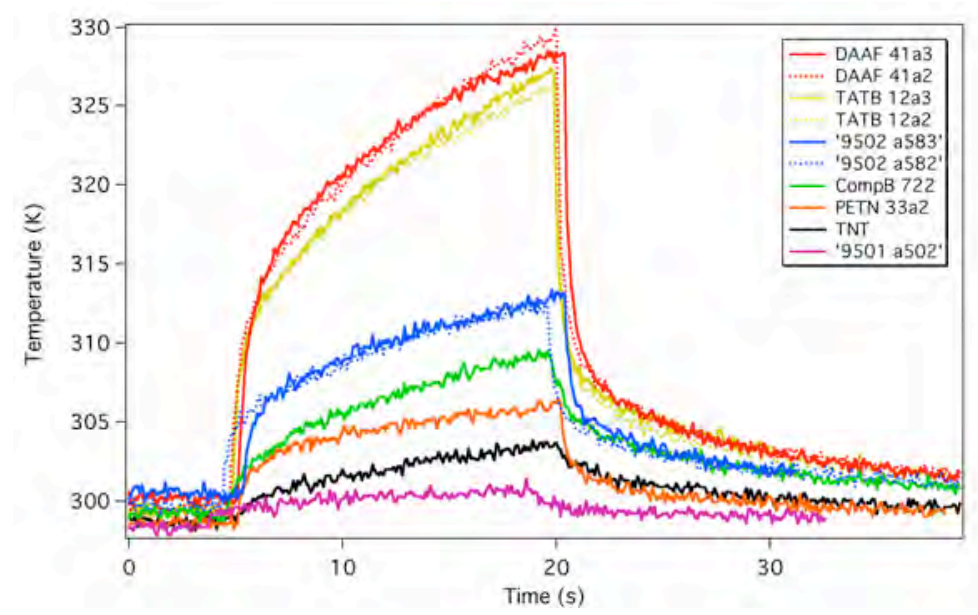

Figure 10. Temperature vs exposure time for several explosives irradiated using a CW $785 \mathrm{~nm}$ laser. None of the materials showed a temperature increase above $330 \mathrm{~K}$ during the 15-second exposure. The reproducibility is shown by the two overlapping curves each for DAAF, TATB, and PBX9502. 
Previous work by Harvey et al. reported the temperature rise of colored materials subjected to $785 \mathrm{~nm}$ laser irradiation. ${ }^{9}$ Their Figure 1 and Table II were utilized to produce a chart of material colors that could produce temperature increases worrisome for energetic materials. The color chart is reproduced in Figure 11. None of the explosives investigated in our study presented in this report have colors on this chart, which is corroborated by the results in Figure 10.

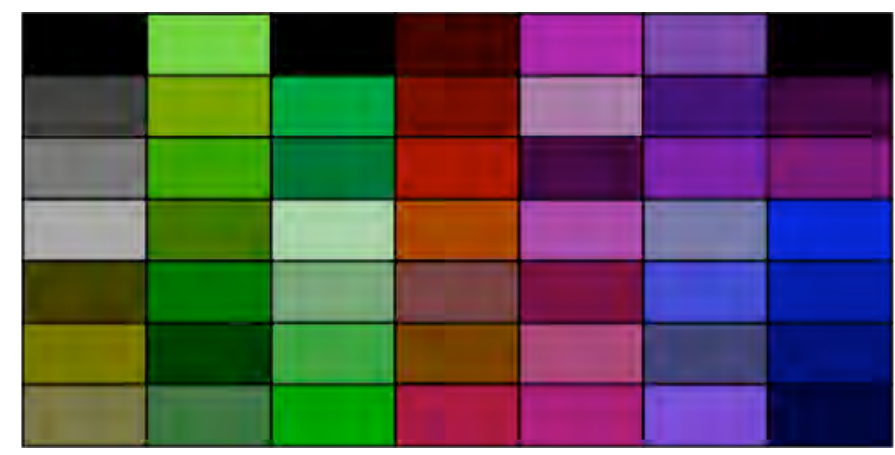

Figure 11. Chart of colors known to absorb $785 \mathrm{~nm}$ laser light sufficiently to cause temperature increases worrisome for energetic materials (extracted from Harvey et al. ${ }^{9}$ ).

\section{Conclusions}

There were no deflagration-to-detonation transitions observed using any of these CW laser wavelengths on single crystals or pressed powders. With unconfined heating, deflagration/flames were observed only under the worst conditions. Except for the photochemistry of DAAF and PBX 9502, all reactions appeared to be thermal using a $532 \mathrm{~nm}$ wavelength laser. For a $1550 \mathrm{~nm}$ wavelength laser, no photochemistry was evident, but the laser power thresholds for some of the materials were significantly lower than for the $532 \mathrm{~nm}$ laser wavelength. Notably, there was no safety concern for single crystals at $532 \mathrm{~nm}$, but at $1550 \mathrm{~nm}$ there does exist a safety concern at high powers (i.e., $>1500 \mathrm{~W} / \mathrm{cm}^{2}$ ).

The temperature vs time data (curves obtained for all samples, but only shown above for $785 \mathrm{~nm}$ in Figure 10 and for single crystals at $532 \mathrm{~nm}$ in Figure 2; data is available as $T, t$ data pairs from the authors), should allow modeling of thermal transport in different geometries. In addition, the thermal properties are dominated by the absorbed energy. This is altered in a complicated manner by multiple scattering events in a powder or pressed explosive. However, for $532 \mathrm{~nm}$ laser irradiation, the visible color of the sample has a high degree of correlation with the heating observed, as illustrated in the aftermath picture shown in Figure 12. Clearly, visible absorption leads to heating by a $532 \mathrm{~nm}$ laser. There is a combination of thermal heating/absorption, reactivity at temperature, and resonant electronic photochemistry that must be considered. The data presented here illustrate that thermal reactions induced by a laser are unlikely to propagate under unconfined conditions and will in all likelihood visibly damage the sample before causing 
a safety concern in contact operations. Nevertheless, these explosives may often react under moderate $532 \mathrm{~nm}$ laser powers in a manner largely predictable from the visible nature of the explosive. Clearly, absorptive inclusions or imperfections may dramatically increase absorption and increase heating rate as well.

At $1550 \mathrm{~nm}$, there appears to be no resonant electronic photochemistry contribution, but the above comments apply equally otherwise.

The available laser power at $785 \mathrm{~nm}$ was insufficient to cause reaction in any of these explosives (pressed pellets), allowing their unrestricted use for investigation using portable Raman spectroscopy devices whose laser powers are less than $300 \mathrm{~mW}$ (all commercial portable Raman systems at present).

We are modeling the thermal response data (spatial and temporal) to resolve the laser spot size vs laser irradiation volume discrepancies caused by the large scattering of the pressed powder samples. The resultant theoretical basis for spot size effects should allow extraction of meaningful laser irradiation parameters for a broad range of explosive materials and experimental conditions.

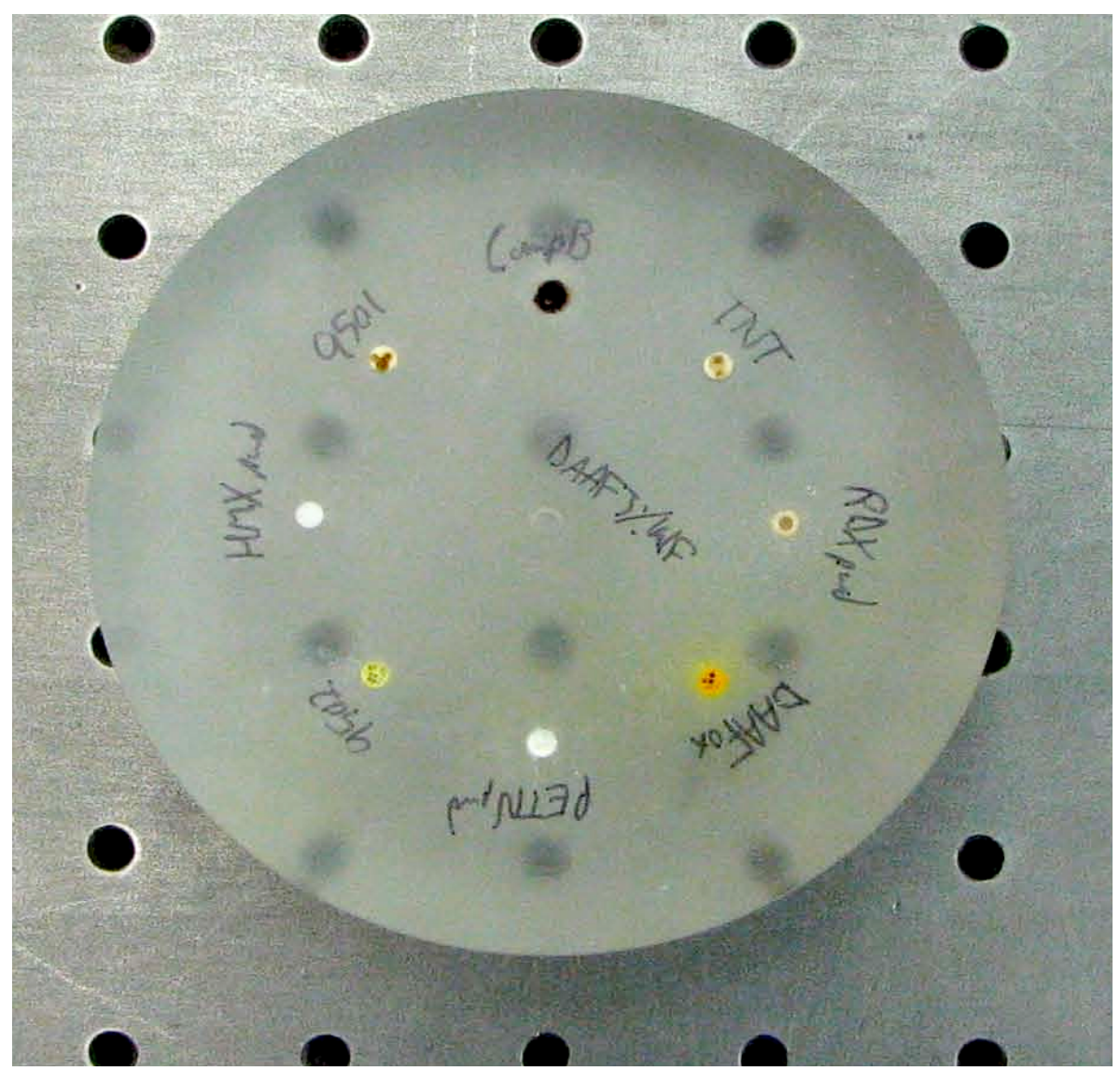

Figure 12. Photograph of $532 \mathrm{~nm}$ wavelength laser irradiated samples. Note color and damage spots. Comp B is essentially all burned but also had significant brown color before the reaction. 


\section{Acknowledgements}

The authors would like to thank Kyle Ramos for the powder samples, Tim Pierce for loan of the fiber laser, Dave Oschwald for loan of the FLIR camera, and Stephanie Hagelberg for pressing the many pellets used in these experiments.

\section{References}

1. H. Östmark, "Laser as a tool in sensitivity testing of explosives," in Proc. Eighth Symposium (International) on Detonation (NSWC, MP 86-194, Silver Spring, MD, 1986), pp. 473-484.

2. P. Dimitriou, V. Hlavacek, S.M. Valone, R.G. Behrens, G.P. Hansen, J.L. Margrave, "Laser-induced ignition in solid-state combustion," AIChE J. 35: 1085-1096 (1989).

3. K.K. Kuo, J.U. Kim, B.L. Fetherolf, T. Torikai, "Preignition dynamics of RDX-based energetic materials under $\mathrm{CO}_{2}$-laser heating," Combust. Flame 95: 351-361 (1993).

4. H. Östmark, M. Carlson, K. Ekvall, "Concentration and temperature measurements in a laser-induced high explosive ignition zone. Part I: LIF spectroscopy measurements," Combust. Flame 105: 381-390 (1996)

5. A.N. Ali, M.M. Sandstrom, D.M. Oschwald, K.M. Moore, S.F. Son, "Laser ignition of DAAF, DHT, and DAATO(3.5)," Propellants, Explos., Pyrotech. 30: 351-355 (2005).

6. A.N. Ali, S.F. Son, B.W. Asay, M.E. Decroix, M.Q. Brewster, "Highirradiance laser ignition of explosives," Combust. Sci. Technol. 175: 15511571 (2003).

7. D. Phillips and P. Wilkins, "Safety test $-1550 \mathrm{~nm}$ laser light on high explosives," HEAF memorandum (May 24, 2010).

8. D.L. Paisley, "Prompt detonation of secondary explosives by laser," in Proc. Ninth Symposium (International) on Detonation (OCNR Vol. 1132910-7, Arlington, VA, 1990) pp. 1110-1117

9. S.D. Harvey, T.J. Peters, and B.W. Wright, "Safety considerations for sample analysis using a near-infrared (785 nm) Raman laser source," Appl. Spectrosc. 57: 580-587 (2003). 
This report has been reproduced directly from the best available copy. It is available electronically on the Web (http://www.doe.gov/bridge).

Copies are available for sale to U.S. Department of Energy employees and contractors from:

Office of Scientific and Technical Information

P.O. Box 62

Oak Ridge, TN 37831

(865) 576-8401

Copies are available for sale to the public from: National Technical Information Service

U.S. Department of Commerce

5285 Port Royal Road

Springfield, VA 22161

(800) 553-6847 
- Los Alamos NATIONAL LABORATORY 